\title{
Mechanisms of RhoGDI2 Mediated Lung Cancer Epithelial-Mesenchymal Transition Suppression
}

\author{
Huiyan Niu Baogang Wu Hongfang Jiang Hui Li Yi Zhang Yang Peng Ping He \\ Department of Geriatrics, Shengjing Hospital, China Medical University, Shenyang, China
}

\author{
Key Words \\ Lung cancer $・$ RhoGDI2 $\bullet$ EMT $・$ Metastasis
}

\begin{abstract}
Background: The aim of this study was to evaluate the function of RhoGDI2 in lung cancer epithelial-mesenchymal transition (EMT) process and to illustrate the underlying mechanisms that will lead to improvement of lung cancer treatment. Methods: The RhoGDI2 knockdown and overexpressing A549 cell lines were first constructed. The influence of RhoGDI2 on cytoskeleton in A549 cells was studied using two approaches: G-LISA-based Rac1 activity measurement and immunostaining-based F-actin distribution. The expression levels of key EMT genes were analyzed using real time quantitative polymerase chain reaction (RT-qPCR), western blot and immunostaining in untreated and RhoGDI2 knock-down or overexpressing A549 cells in both in vivo and in vitro experimental settings. Results: Our study showed that the activity of Rac1, a key gene that is crucial for the initiation and metastasis of human lung adenocarcinoma, causing the redistribution of F-actin with partial loss of cell-cell adhesions and stress fibers, was significantly suppressed by RhoGDI2. RhoGDI2 promoted the expression of EMT marker gene E-cadherin and repressed EMT promoting genes Slug, Snail, $\alpha$-SMA in both A549 cells and lung and liver organs derived from the mouse models. Knocking-down RhoGDI2 induced abnormal morphology for lung organs. Conclusion: These findings indicate that RhoGDI2 repressed the activity of Rac1 and may be involved in the rearrangement of cytoskeleton in lung cancer cells. RhoGDI2 suppresses the metastasis of lung cancer mediated through EMT by regulating the expression of key genes such as E-cadherin, Slug, Snail and $\alpha$-SMA in both in vivo and in vitro models.
\end{abstract}




\section{Introduction}

Lung cancer is the number one cause of cancer mortality, killing more people than the total number of deaths caused by colon, pancreatic, breast and prostate cancers [1]. The prognosis for advanced lung cancer patient is very poor [2-4], which is often associated with epithelial-mesenchymal transition (EMT) mediated metastasis [5-8].

EMT was initially studied as a process during embryonic development in which epithelial cells emerge from their original niche and migrate to distal regions [6, 7, 9-12]. Recently more and more studies have suggested that EMT is a crucial process during cancer cells' invasion and metastasis in which epithelial cells down-regulate epithelial adherens and tight junction proteins, lose apical-basal polarity and cell-cell contacts, and undergo remarkable remodeling of the cytoskeleton to facilitate cell motility and invasion $[6,9-11,13,14]$.

Rho GDP dissociation inhibitors (RhoGDIs), including three members, RhoGDI1, RhoGDI2 and RhoGDI3, are important regulators of Rho GTPases activity through proteinprotein interaction [15-18]. One of the key functions of Rho GTPases is to remodel cytoskeleton components thus influence cell mobility. Therefore, it is not surprising that regulation of Rho family proteins can have profound impact on metastasis, a process that involves cancer cells migrating from one location to another and invading the destination tissue/organ [19]. RhoGDI2 is mainly expressed in hematopoietic cells and is differentially expressed in multiple cancers [20-22]. The roles of RhoGDI2 in regulating cancer cell growth and metastasis vary in different cancers. On the one hand, RhoGDI2 promotes the growth and invasion behavior for gastric, ovarian and breast cancers [23-26]. On the other hand, RhoGDI2 represses metastasis of bladder cancer and mouse lung cancer [19, 27-30]. However, the detailed mechanism by which RhoGDI2 regulates lung cancer EMT process is still elusive. Here, we report our work to demonstrate the roles played by RhoGDI2 in mediating the EMT process in lung cancer, through the use of an in vivo EMT model and in vitro protein chemistry.

\section{Materials and Methods}

Cell lines and antibodies

Lung cancer cell line A549 was purchased from Shanghai Institute of Biochemistry and Cell Biology, Chinese Academy of Sciences. The following antibodies were used: human anti-E-cadherin antibody (Santa Cruz Biotechnology), human anti-Slug antibody (Santa Cruz Biotechnology), human anti-Snail antibody (Santa Cruz Biotechnology), human anti- $\alpha$-SMA antibody (Abcam), anti-F-actin (Abcam), and anti- $\beta$-actin (Santa Cruz Biotechnology).

Cell culture, gene knockdown and overexpression

A549 cells were cultured in RPMI-1640 medium containing 10\% fetal bovine serum, $100 \mathrm{IU} / \mathrm{ml}$ penicillin and $100 \mu \mathrm{g} / \mathrm{ml}$ streptomycin. The cells were grown in a humidified incubator at $37{ }^{\circ} \mathrm{C}$ and under an atmosphere of $5 \% \mathrm{CO}_{2}$. Cells were grown on sterile tissue culture petri dishes and passaged once every 2 to 3 days $[19,31,32]$.

Stable knockdown of RhoGDI2 was achieved by transfecting cells with shRNAs (5' CCCCGCTCAATTATAAGCCTCCATTCAAGAGATGGAGGCTTATAATTGAGCTTTTT $33^{\prime}$ and 5' AAAGCTCAATTATAAGCCTCCATCTCTTGAATGGAGGCTTATAATTGAGCGGG 3') -carrying lentivirus and by keeping cells cultured in selection media for 2 weeks. Cells with the most efficient knockdown were collected for subsequent experiments. Full-length RhoGDI2 was cloned into a pIRES2-EGFP vector (Invitrogen). The pIRES2-EGFP-RhoGDI2 or the empty vector was transfected into cells using Lipofectamine 2000 transfection reagent (Invitrogen) according to the manufacturer's recommendations.

Rac1 activity assay

The activity of Rac1 (GTP bound form) was quantified using G-LISA Rac1 activation assay Biochem Kit (Cytoskeleton) according to the manufacturer's instructions. Briefly, a total of $180 \mu$ l protein extract 
was added to each corresponding well pre-coated with Rac-GTP-binding protein, with $36 \mu$ Rac1 used as positive control. These were put on ice for $30 \mathrm{~min}$ followed by incubation with $20 \mu \mathrm{l}$ of anti-Rac1 for 30 min. Subsequently, horseradish peroxidase (HRP)-conjugated secondary antibody (50 $\mu$ l) was added to each well and incubation was continued for $45 \mathrm{~min}$ on ice. Subsequently, $50 \mu \mathrm{l}$ HRP detection reagent was incubated for $20 \mathrm{~min}$ at room temperature. The reaction was stopped by adding $50 \mu \mathrm{HRP}$ stop solution and the absorbance was recorded at $490 \mathrm{~nm}$ (Biotek Instruments, Winsooki, VT). The activity of Rac1 was computed according to the manufacturer's recommendation.

\section{$R T-q P C R$}

Total RNA was extracted from all samples using TRIZOL reagent (Invitrogen) according to manufacturer's instructions, then reverse-transcribed using SuperScript First Strand cDNA System (Invitrogen) according to the manufacturer's instructions. qPCR was performed using Exicycler 96 (BIONEER) following manufacturer's advice. The sequences of the PCR primers are: E-cadherin-upstream (5' ATGCCGCCATCGCTTACAC 3'), E-cadherin-downstream (5' CGACGTTAGCCTCGTTCTCA 3'), Slug-upstream (5' AGCGAACTGGACACACATAC 3'), Slug-downstream (5' GCCCCAAAGATGAGGAGTAT 3'), Snail-upstream (5' GCCCCACAGGACTTTGATGA 3'), Snail-downstream (5' AGTGAGTCTGTCAGCCTTTGTC 3'), $\alpha$-SMA-upstream (5' TCCCTTGAGAAGAGTTACGAGTT 3'), $\alpha$-SMA-downstream (5' ATGATGCTGTTGTAGGTGGTT 3'), $\beta$-actinupstream(5'CTTAGTTGCGTTACACCCTTTCTTG3'), $\beta$-actin-downstream(5'CTGTCACCTTCACCGTTCCAGTTT 3').

\section{Western blot}

Western blot was performed using standard technique. Briefly cells were lysed in RIPA buffer ( $20 \mathrm{mM}$ Tris-HCl, pH 7.4, 20\% glycerol, 0.5\% NP-40, 1 mM MgCl $2^{\prime} 0.5$ M NaCl, 1 mM EDTA, 1 mM EGTA, and aprotinin), and separated by sodium dodecyl sulfate-polyacrylamide gel electrophoresis (SDS-PAGE), transferred to polyvinylidene difluoride (PVDF) membrane, incubated for $2 \mathrm{~h}$ with antibodies in TBST containing 5\% non-fat dried milk at room temperature. After incubation with appropriate horseradish peroxidase (HRP)conjugated secondary antibodies, the signals were detected using ECL Western Blotting Detection Kit (BD Bioscience) after washing.

\section{Immunofluorescence}

Cells growing on the coverslips were washed briefly and fixed in 4\% paraformaldehyde in PBS for 10 min on ice. After permeabilization in PBS containing $0.1 \%$ Triton X-100 on ice for 10 minutes, cells were washed using PBS for three times. After blocking in $5 \%$ milk for 30 min at $37{ }^{\circ} \mathrm{C}$, anti-F-actin, and Cy3-conjugated anti rabit IgG (Beyotime) antibodies were added with a dilution ratio as suggested by the manufacturer. Cells were then counterstained with DAPI (Biosharp). Fluorescent images were acquired using OLYMPUS IX51 confocal microscope after washing off the unbound secondary antibodies. The same procedure was used for cell staining by antibodies against E-cadherin, Slug, Snail and $\alpha$-SMA.

\section{Mouse model for tumor metastasis}

Thirty healthy male mice with severe combined immunodeficiency (SCID) at the age 5 weeks post birth were selected. Each one of them weighted $\sim 20 \mathrm{~g}$. These mice were randomly assigned to 5 groups and each group was injected with untreated A549 cells, control knock-down A549 cells, RhoGDI2 knock-down A549 cells, control overexpression A549 cells and RhoGDI2 overexpressing A549 cells at a density of $2 \times 10^{5}$ $/ 200 \mu \mathrm{l}$, respectively. After 8 weeks, the lung and liver organs of the animals were harvested.

\section{Immunohistochemistry}

Immunohistochemical staining was performed on paraffin-embedded lung and liver organ specimens. Slides were routinely deparaffinized and hydrated. Subsequently, the sections were incubated in hematoxylin (Solarbio) for $5 \mathrm{~min}$. After normal washing and soaking, the sections were counterstained with eosin for 3-5 min. After dehydration, clarification and sealing processes, the slides were imaged under DP73 (OLYMPUS).

\section{Statistical analysis}

Student's t-test was used for all statistical analyses for experimental results. All statistical calculations were performed using R software. A p-value $<0.05$ was considered statistically significant. 
Fig. 1. RhoGDI2 affects the distribution of cytoskeleton. (A) Activity of Rac1 in untreated and RhoGDI2 knock-down and overexpressing A549 cells. '*' represents $\mathrm{p}<0.05$ by Student's t-test. (B) Localization of F-actin in untreated and RhoGDI2 knock-down and overexpressing A549 cells.

\section{Results}

\section{RhoGDI2 rearranges cytoskeleton}

We first checked whether RhoGDI2 would affect the cytoskeleton of A549 cells. Rac1 is an important GTPase in regulating cytoskeleton organization, cell adhesion and transcriptional activation [33, 34]. Silencing Rac1 reduced the migration and invasion of lung cancer which was accompanied by concomitant cytoskeleton rearrangements [35]. Knock-down RhoGDI2 significantly increased Rac1 activity in A549 cells, whereas overexpressing RhoGDI2 significantly decreased the activity of Rac1 (Fig. 1A), indicating RhoGDI2 may repress the function of Rac1. Further, F-actin filaments became scattered throughout the cytoplasm and stress fibers were lost in RhoGDI2 knock-down A549 cells compared with control cells (Fig. 1B). Taken together, these results suggested that RhoGDI2 represses Rac1 activity, which in turn disrupts the redistribution of cytoskeleton during EMT.

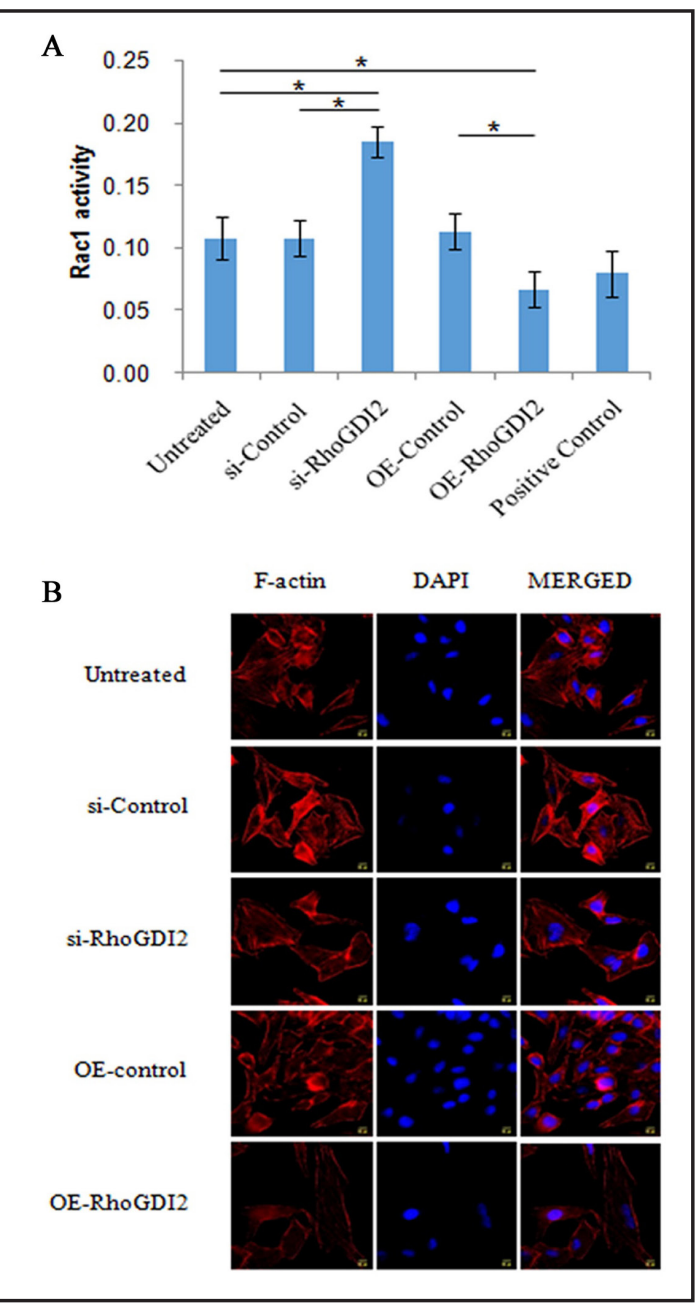

RhoGDI2 promotes the expression of E-cadherin and represses Slug, Snail and $\alpha$-SMA

Down-regulation of E-cadherin, a repressor of metastasis, is considered as the hallmark of EMT in cancer [36-38]. Several transcription factors such as Slug, Snail, are normally considered repressors for E-cadherin [36, 39-41]. In A549 cells, the knockdown of RhoGDI2 significantly decreased the expression of E-cadherin, whereas overexpressing RhoGDI2 increased the expression of E-cadherin (Fig. 2A and Fig. 3A, B). Conversely, knocking down RhoGDI2 promoted the expression of Slug, Snail and $\alpha$-SMA while overexpressing RhoGDI2 repressed their expression (Fig. 2B-D and Fig. 3A, B). In combination, these results indicated that RhoGDI2 may regulate the expression of key EMT genes to effect the EMT process in A549 cells.

The morphology of lung and liver tissues from in vivo EMT model

To check the influence of RhoGDI2 on the morphology of lung and liver tissues, we stained lung and liver organs with hematoxylin and eosin. As expected, lungs extracted from mice injected with RhoGDI2 knock-down A549 cells showed significantly increased level of tumorigenesis, and those from mice injected with RhoGDI2 overexpressing A549 cells exhibited similar phenotypes to those of the control mice (Fig. 4). However, liver organs harvested from mice injected with either RhoGDI2 knock-down or overexpressing A549 cells showed no visible morphological changes from those of the control mice (Fig. 4). Taken together, these results provided evidence that RhoGDI2 may suppresses tumorigenesis in lung cells. 
Fig. 2. RhoGDI2 regulates the intracellular distribution of key EMT genes. Localization of E-cadherin (A), Slug (B), Snail (C) and $\alpha$-SMA (D) in untreated and RhoGDI2 knockdown and overexpressing A549 cells were examined by immunostaining.

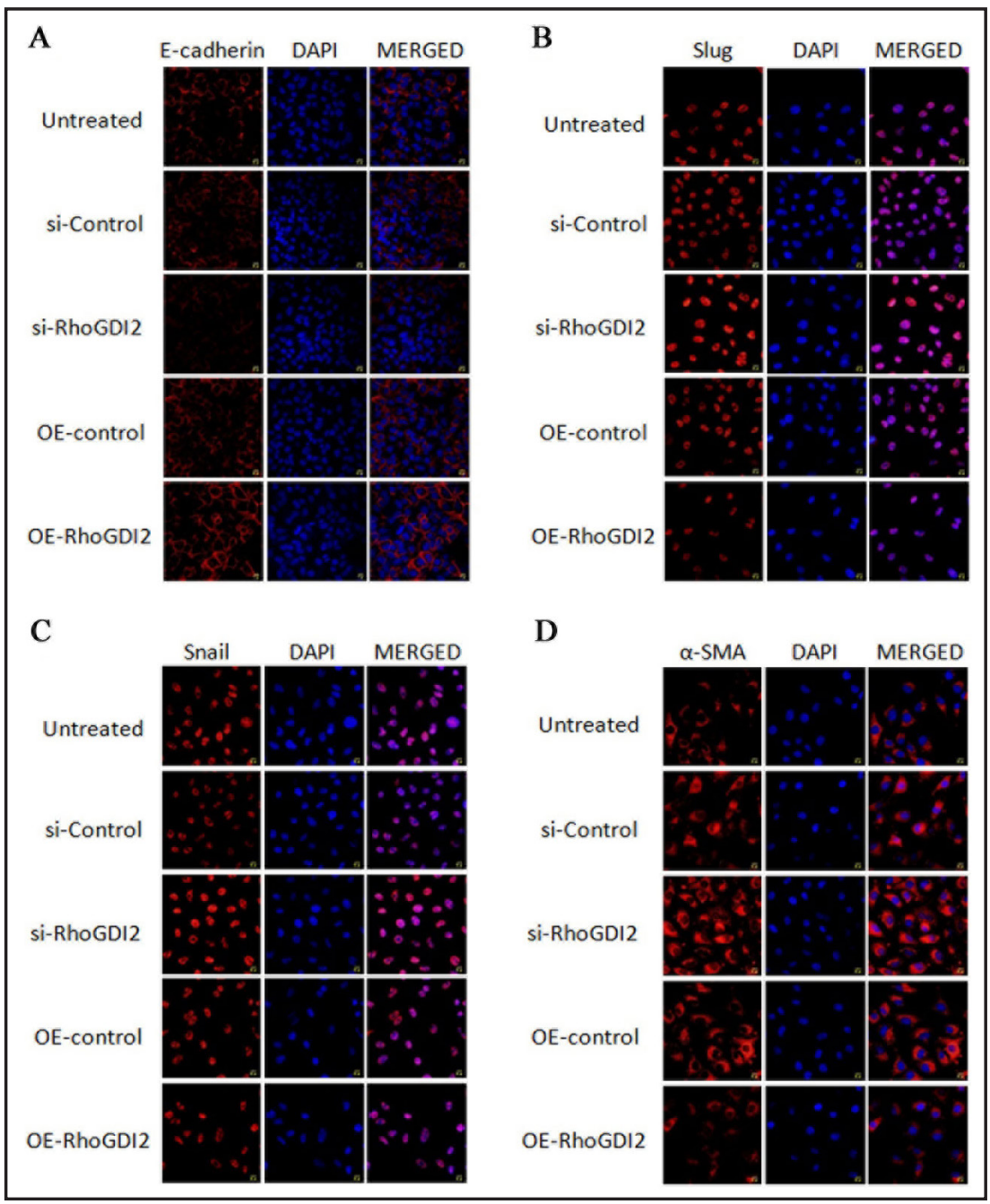

RhoGDI2 exhibits similar regulatory roles in cancer development in vivo as it does in vitro

To investigate the in vivo mechanism underlying RhoGDI2 repression of lung cancer metastasis, we checked the expression levels of key genes involved in the epithelialmesenchymal transition (EMT) process. Similar to the results obtained in vitro (Fig. 3A-B), RhoGDI2 promoted the expression of E-cadherin and repressed the expression of Slug, Snail and $\alpha$-SMA in both lung and liver organs (Fig. 5A-B and Fig. 6A-B). These results suggested that RhoGDI2 may regulate the expression of key genes involved in the EMT process.

\section{Discussion}

Despite recent advances in tumor-treatment technologies, lung cancer is still a top killer in all tumor-related deaths. Tumor metastasis is one of the major causes for lung cancer related death. RhoGDI2 is encoded by the ARHGDIB gene and is differentially expressed in various tissues in human [42]. While there are clear links between the alteration of RhoGDI2 protein levels and disease progression and/or the metastasis status in several types of cancer, the role that RhoGDI2 played in regulating cancer cell growth and metastasis varied in different types of cancers. For example, RhoGDI2 suppresses invasion and metastasis in bladder cancer and mouse lung cancer[19, 27-29] and is a positive prognostic factor [42]. Moreover, RhoGDI2 is selectively down-regulated in Hodgkin lymphoma cells compared with non-Hodgkin lymphoma [43]. But for gastric, ovarian and breast cancers, RhoGDI2 promoted 
Fig. 3. RhoGDI2 affects the expression level of key EMT genes. The changes in the expression levels of E-cadherin, Slug, Snail and $\alpha$-SMA were examined by RT-qPCR (A) and western blot (B) in untreated and RhoGDI2 knock-down and overexpressing A549 cells. '*' represents $\mathrm{p}<$ 0.05 by Student's t-test.

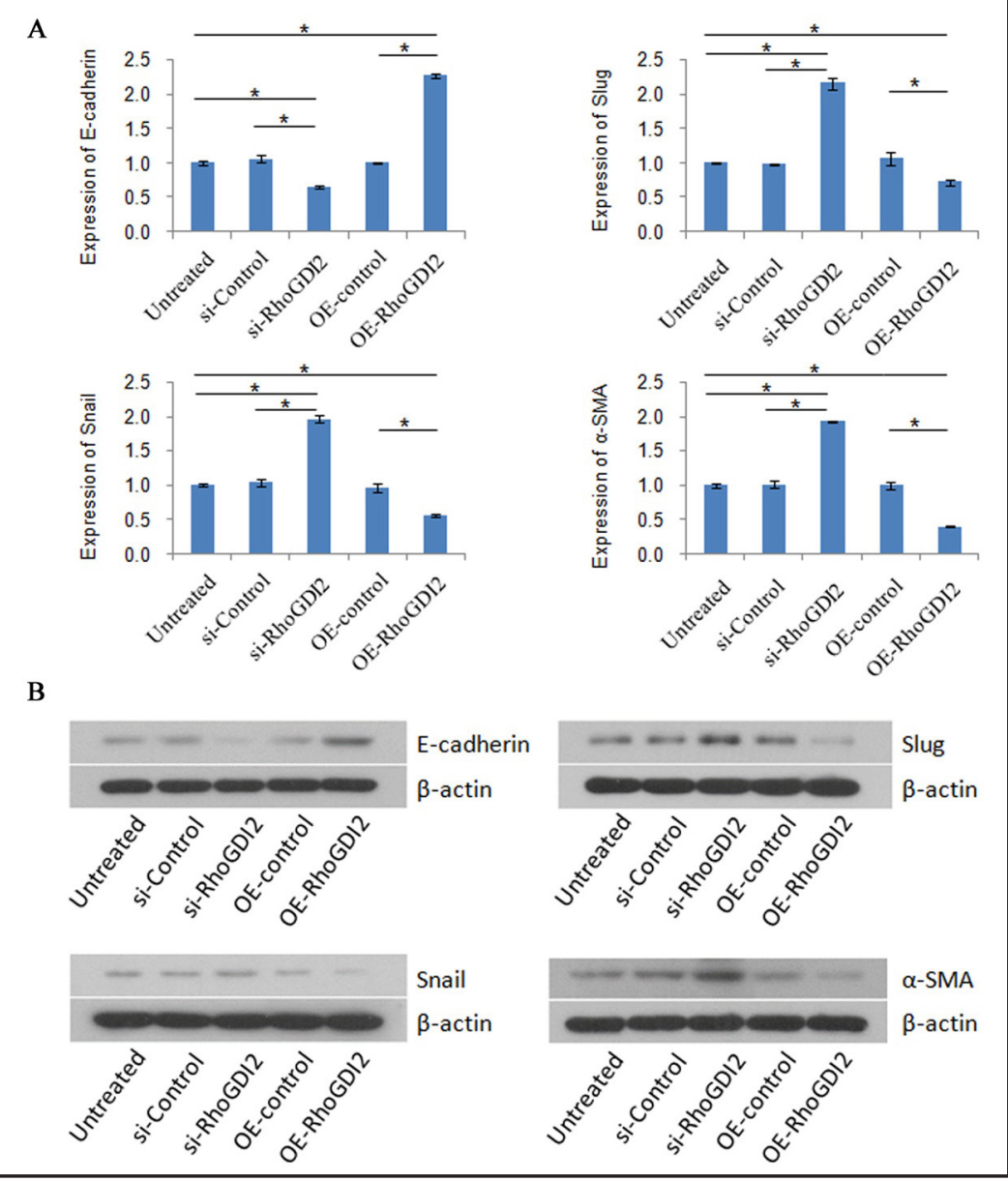

Fig. 4. RhoGDI2 influences organ morphology resulted from cancer development. Vertical panels are immunohistochemical staining showing the morphological changes of lung and liver organs in mouse models injected with untreated and RhoGDI2 knockdown and overexpressing A549 cells.

their growth and invasion behavior [2326]. Therefore it is necessary to explore the underlying mechanism to explain the variety of functions that RhoGDI2 plays in different cancers. In previous studies, we found that the expression of RhoGDI2 protein was lower in lung cancer tissues than in normal lung tissues, and that RhoGDI2 may interact with the PI3K/Akt pathway [19]. Based on our data, we took lung cancer as a model system to study the potential roles that RhoGDI2 play in lung cancer EMT process and to illuminate the underlying mechanisms.

As a molecular switch for the organization and dynamics of the actin cytoskeleton, Rac-1 was found to be bound

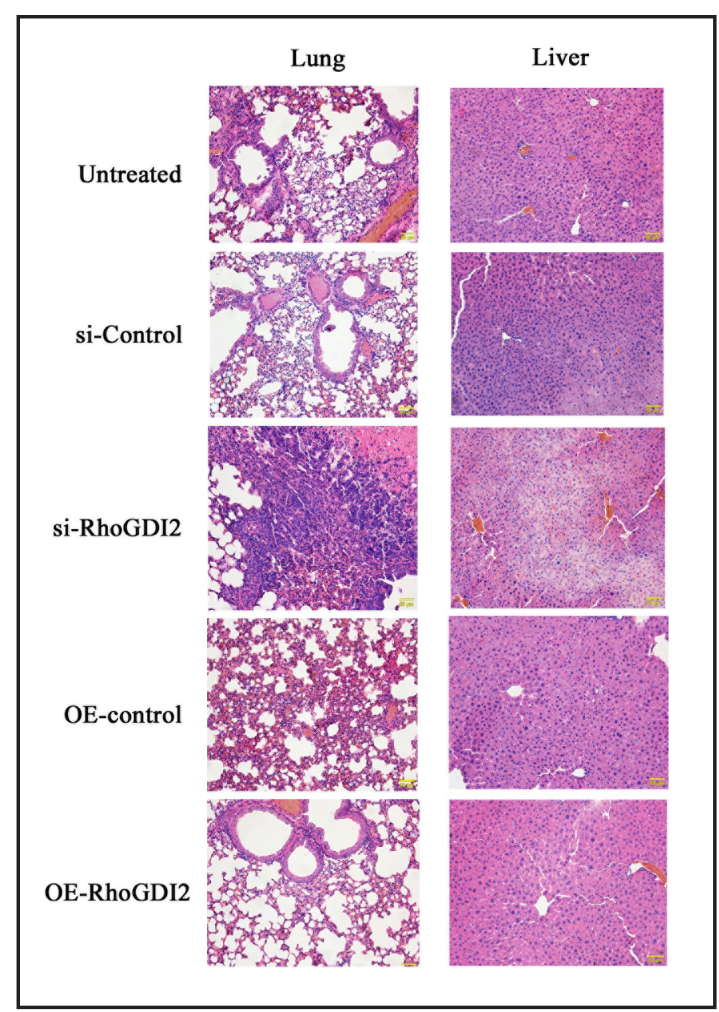


Fig. 5. RhoGDI2 affects the expression of key EMT genes in lung tissue of metastasis mouse models. The changes in the expression levels of E-cadherin, Slug, Snail and $\alpha$-SMA were detected by RT-qPCR (A) and western blot (B) in lungs jected with untreated and RhoGDI2 knock-down and overexpressing A549 cells. '*' represents $p<0.05$ by Student's t-test. harvested from mice in-

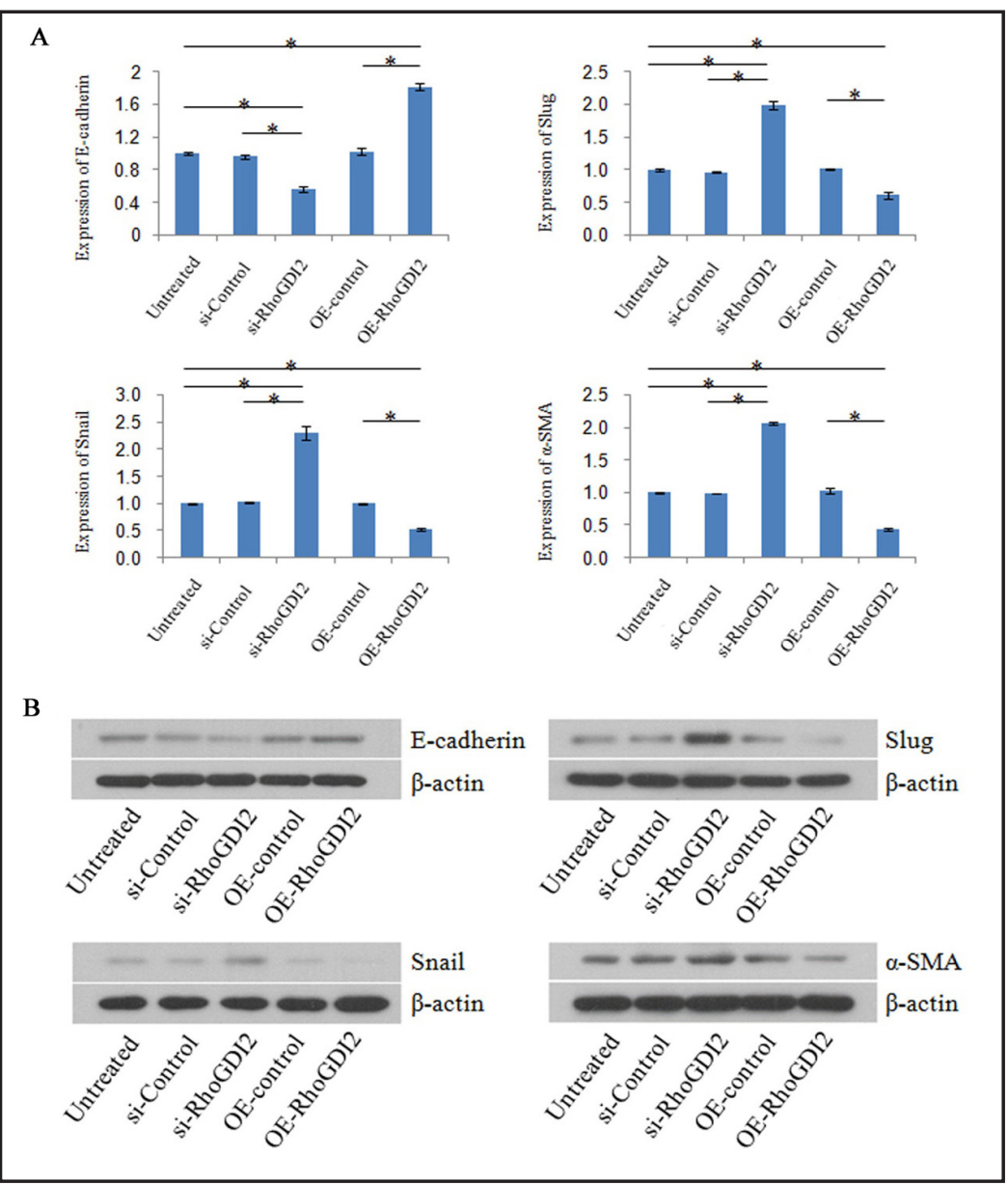

and activated by RhoGDI2 in bladder cancer[44], whereas this interaction inactivates Rac1 in breast and gastric cancer[23, 45]. Our results showed that RhoGDI2 repressed the activity of Rac1, which is evidenced by the redistribution of F-actin with partial loss of cellcell adhesions and stress fibers, and possibly the rearrangement of cytoskeleton in A549 cells. This further strengthens the notion that RhoGDI2 may play opposite roles in regulating cancer metastasis depending on cell types and cellular environment.

Epithelial-mesenchymal transition (EMT) is considered a crucial process for initiating cancer metastasis $[6,9-11,13,14]$. During tumor EMT, epithelial tumor cells detach from their origin, obtain fibroblastic characteristics and migrate to distal regions to start the invasion process. E-cadherin is a cell-cell adhesion glycoprotein. Its expression is crucial for establishing and maintaining epithelial tissue structure [46]. Down-regulation of E-cadherin decreases cell adhesion strength and promotes motility, hence it is considered the fundamental hallmark of EMT in cancer [5, 6, 36-38]. It is widely accepted that E-cadherin represses cell invasion and metastasis $[5,6,37,38]$, and its activity can be regulated by several transcription factors e.g., Slug and Snail especially in lung carcinoma [36, 39-41]. Down-regulation of E-cadherin is usually caused by the expression of transcription factors such as Slug, Snail, Zeb1, E47 [36]. To better elucidate the role of RhoGDI2 in metastasis and cancer cell invasion, the effect of RhoGDI2 overexpression or depletion on EMT was investigated. As expected, the expression of E-cadherin, the hallmark and repressor of EMT process, was promoted by RhoGDI2 overexpression in A549 cells lines as well as in the lung and liver organs segregated from tumor metastasis mouse models. Accordingly, the expression of Slug, Snail and $\alpha$-SMA was repressed by RhoGDI2 in both A549 cell lines and in vivo model. Animal experiments showed that knock-down RhoGDI2 did promote the metastasis of lung cancer. 
Fig. 6. RhoGDI2 affects the expression of key EMT genes in liver tissue of metastasis mouse models. The changes in the expression levels of E-cadherin, Slug, Snail and $\alpha$-SMA were detected by RT-qPCR (A) and western blot (B) in liver organs harvested from mouse models injected with untreated and RhoGDI2 knockdown and overexpressing A549 cells. '*' represents p < 0.05 by Student's t-test.

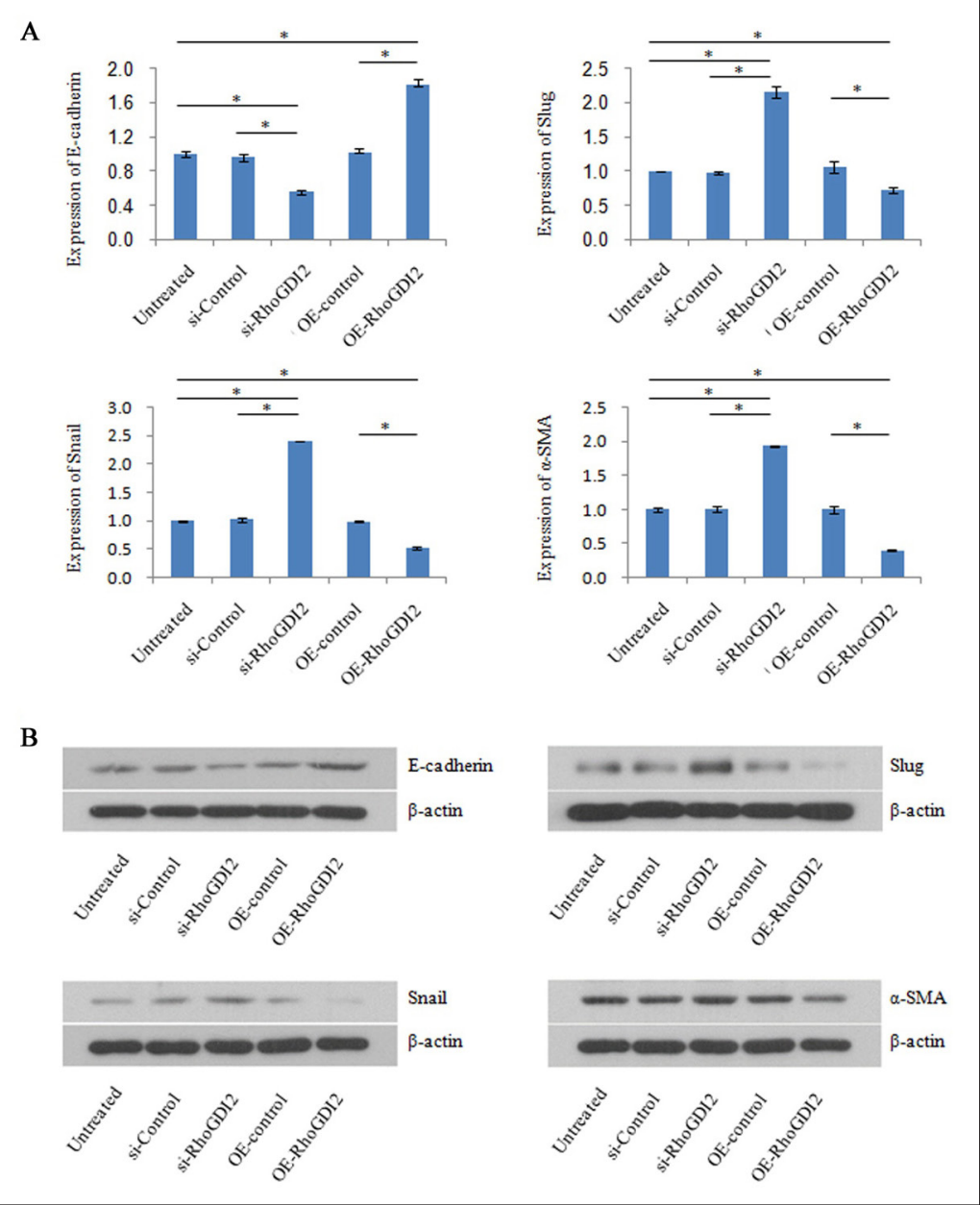

A

B

Though the changes in the expression levels of key genes like E-cadherin, Slug, Snail and $\alpha$-SMA were similar in lung and liver organs derived from tumor metastasis model in response to altered the expression of RhoGDI2, the number of nodules formed and the morphological changes in lung and liver were different. This suggests that there may exist other factors that are also involved in RhoGDI2-mediated EMT process whose roles are organ-specific roles. Further experiments are needed to shed more light on this phenomenon and to identify the direct down-stream targets of RhoGDI2 signaling.

Our work suggested that RhoGDI2 suppressed lung cancer EMT process and metastasis in vitro and in vivo by molecular mark detection and organ morphology characterization. We also found that the roles RhoGDI2 played in lung cancer metastasis were partly through altering cytoskeleton and by regulating the expression of key EMT genes. Our work hinted the potential of RhoGDI2 to serve as a therapy target for advanced lung cancer.

\section{Disclosure Statement}

The authors declare no competing interests.

\section{Acknowledgments}

This work was supported by a grant from the National Natural Science Foundation of China (No.81201832) and Specialized Research, Fund for the Doctoral Program of Higher Education (No. 20122104110011). 
Niu et al.: RhoGDI2 Suppresses EMT in Lung Cancer

\section{References}

1 Ferlay J, Parkin DM, Steliarova-Foucher E: Estimates of cancer incidence and mortality in europe in 2008. Eur J Cancer 2010;46:765-781.

2 Stallings-Mann ML, Waldmann J, Zhang Y, Miller E, Gauthier ML, Visscher DW, Downey GP, Radisky ES, Fields AP, Radisky DC: Matrix metalloproteinase induction of rac1b, a key effector of lung cancer progression. Sci Transl Med 2012;4:142ra195.

-3 Cufer T, Ovcaricek T, O'Brien ME: Systemic therapy of advanced non-small cell lung cancer: Majordevelopments of the last 5-years. Eur J Cancer 2013;49:1216-1225. Spira A, Ettinger DS: Multidisciplinary management of lung cancer. N Engl J Med 2004;350:379-392. Chaffer CL, Weinberg RA: A perspective on cancer cell metastasis. Science 2011;331:1559-1564. Kalluri R, Weinberg RA: The basics of epithelial-mesenchymal transition. J Clin Invest 2009;119:14201428.

7 Micalizzi DS, Farabaugh SM, Ford HL: Epithelial-mesenchymal transition in cancer: Parallels between normal development and tumor progression. J Mammary Gland Biol Neoplasia 2010;15:117-134.

8 Hess KR, Varadhachary GR, Taylor SH, Wei W, Raber MN, Lenzi R, Abbruzzese JL: Metastatic patterns in adenocarcinoma. Cancer 2006;106:1624-1633.

9 Yang J, Weinberg RA: Epithelial-mesenchymal transition: At the crossroads of development and tumor metastasis. Dev Cell 2008;14:818-829.

10 Thiery JP, Acloque H, Huang RY, Nieto MA: Epithelial-mesenchymal transitions in development and disease. Cell 2009;139:871-890.

11 Nieto MA: Epithelial-mesenchymal transitions in development and disease: Old views and new perspectives. Int J Dev Biol 2009;53:1541-1547.

12 Nakaya Y, Sheng G: Emt in developmental morphogenesis. Cancer Lett 2013;341:9-15.

13 Acloque H, Adams MS, Fishwick K, Bronner-Fraser M, Nieto MA: Epithelial-mesenchymal transitions: The importance of changing cell state in development and disease. J Clin Invest 2009;119:1438-1449.

14 Kong D, Li Y, Wang Z, Sarkar FH: Cancer stem cells and epithelial-to-mesenchymal transition (emt)phenotypic cells: Are they cousins or twins? Cancers (Basel) 2011;3:716-729.

15 Dovas A, Couchman JR: Rhogdi: Multiple functions in the regulation of rho family gtpase activities. Biochem J 2005;390:1-9.

16 DerMardirossian C, Bokoch GM: Gdis: Central regulatory molecules in rho gtpase activation. Trends Cell Biol 2005;15:356-363.

17 Hart MJ, Maru Y, Leonard D, Witte ON, Evans T, Cerione RA: A gdp dissociation inhibitor that serves as a gtpase inhibitor for the ras-like protein cdc42hs. Science 1992;258:812-815.

18 Chuang TH, Xu X, Knaus UG, Hart MJ, Bokoch GM: Gdp dissociation inhibitor prevents intrinsic and gtpase activating protein-stimulated gtp hydrolysis by the rac gtp-binding protein. J Biol Chem 1993;268:775-778.

19 Niu H, Li H, Xu C, He P: Expression profile of rhogdi2 in lung cancers and role of rhogdi2 in lung cancer metastasis. Oncol Rep 2010;24:465-471.

20 Cho HJ, Baek KE, Yoo J: Rhogdi2 as a therapeutic target in cancer. Expert Opin Ther Targets 2010;14:67-75.

-21 Scherle P, Behrens T, Staudt LM: Ly-gdi, a gdp-dissociation inhibitor of the rhoa gtp-binding protein, is expressed preferentially in lymphocytes. Proc Natl Acad Sci U S A 1993;90:7568-7572.

22 Griner EM, Theodorescu D: The faces and friends of rhogdi2. Cancer Metastasis Rev 2012;31:519-528.

23 Cho HJ, Park SM, Kim IK, Nam IK, Baek KE, Im MJ, Yoo JM, Park SH, Ryu KJ, Han HT, Kim HJ, Hong SC, Kim KD, Pak Y, Kim JW, Lee CW, Yoo J: Rhogdi2 promotes epithelial-mesenchymal transition via induction of snail in gastric cancer cells. Oncotarget 2014;5:1554-1564.

24 Tapper J, Kettunen E, El-Rifai W, Seppala M, Andersson LC, Knuutila S: Changes in gene expression during progression of ovarian carcinoma. Cancer Genet Cytogenet 2001;128:1-6.

25 Zhang Y, Zhang B: D4-gdi, a rho gtpase regulator, promotes breast cancer cell invasiveness. Cancer Res 2006;66:5592-5598.

26 Cho HJ, Baek KE, Park SM, Kim IK, Choi YL, Cho HJ, Nam IK, Hwang EM, Park JY, Han JY, Kang SS, Kim DC, Lee WS, Lee MN, Oh GT, Kim JW, Lee CW, Yoo J: Rhogdi2 expression is associated with tumor growth and malignant progression of gastric cancer. Clin Cancer Res 2009;15:2612-2619. 
Niu et al.: RhoGDI2 Suppresses EMT in Lung Cancer

-27 Gildea JJ, Seraj MJ, Oxford G, Harding MA, Hampton GM, Moskaluk CA, Frierson HF, Conaway MR, Theodorescu D: Rhogdi2 is an invasion and metastasis suppressor gene in human cancer. Cancer Res 2002;62:6418-6423.

-28 Titus B, Frierson HF, Jr., Conaway M, Ching K, Guise T, Chirgwin J, Hampton G, Theodorescu D: Endothelin axis is a target of the lung metastasis suppressor gene rhogdi2. Cancer Res 2005;65:7320-7327.

29 Said N, Sanchez-Carbayo M, Smith SC, Theodorescu D: Rhogdi2 suppresses lung metastasis in mice by reducing tumor versican expression and macrophage infiltration. J Clin Invest 2012;122:1503-1518.

-30 Agarwal NK, Chen CH, Cho H, Boulbes DR, Spooner E, Sarbassov DD: Rictor regulates cell migration by suppressing rhogdi2. Oncogene 2013;32:2521-2526.

-31 Niu H, Wang J, Li H, He P: Rapamycin potentiates cytotoxicity by docetaxel possibly through downregulation of survivin in lung cancer cells. J Exp Clin Cancer Res 2011;30:28.

-32 Niu H, Zhang Y, Wu B, Jiang H, He P: Matrine induces the apoptosis of lung cancer cells through downregulation of inhibitor of apoptosis proteins and the akt signaling pathway. Oncol Rep 2014;32;10871093.

33 Price LS, Collard JG: Regulation of the cytoskeleton by rho-family gtpases: Implications for tumour cell invasion. Semin Cancer Biol 2001;11:167-173.

34 Schmitz AA, Govek EE, Bottner B, Van Aelst L: Rho gtpases: Signaling, migration, and invasion. Exp Cell Res 2000;261:1-12.

35 Chen QY, Xu LQ, Jiao DM, Yao QH, Wang YY, Hu HZ, Wu YQ, Song J, Yan J, Wu LJ: Silencing of rac1 modifies lung cancer cell migration, invasion and actin cytoskeleton rearrangements and enhances chemosensitivity to antitumor drugs. Int J Mol Med 2011;28:769-776.

-36 Lim SC, Jang IG, Kim YC, Park KO: The role of e-cadherin expression in non-small cell lung cancer. J Korean Med Sci 2000;15:501-506.

37 Bae GY, Choi SJ, Lee JS, Jo J, Lee J, Kim J, Cha HJ: Loss of e-cadherin activates egfr-mek/erk signaling, which promotes invasion via the zeb1/mmp2 axis in non-small cell lung cancer. Oncotarget 2013;4:2512-2522.

-38 Kase S, Sugio K, Yamazaki K, Okamoto T, Yano T, Sugimachi K: Expression of e-cadherin and beta-catenin in human non-small cell lung cancer and the clinical significance. Clin Cancer Res 2000;6:4789-4796.

-39 Shih JY, Tsai MF, Chang TH, Chang YL, Yuan A, Yu CJ, Lin SB, Liou GY, Lee ML, Chen JJ, Hong TM, Yang SC, Su JL, Lee YC, Yang PC: Transcription repressor slug promotes carcinoma invasion and predicts outcome of patients with lung adenocarcinoma. Clin Cancer Res 2005;11:8070-8078.

40 Yanagawa J, Walser TC, Zhu LX, Hong L, Fishbein MC, Mah V, Chia D, Goodglick L, Elashoff DA, Luo J, Magyar CE, Dohadwala M, Lee JM, St John MA, Strieter RM, Sharma S, Dubinett SM: Snail promotes cxcr2 liganddependent tumor progression in non-small cell lung carcinoma. Clin Cancer Res 2009;15:6820-6829.

41 Merikallio H, Turpeenniemi-Hujanen T, Paakko P, Makitaro R, Riitta K, Salo S, Salo T, Harju T, Soini Y: Snail promotes an invasive phenotype in lung carcinoma. Respir Res 2012;13:104.

42 Theodorescu D, Sapinoso LM, Conaway MR, Oxford G, Hampton GM, Frierson HF Jr: Reduced expression of metastasis suppressor rhogdi2 is associated with decreased survival for patients with bladder cancer. Clin Cancer Res 2004;10:3800-3806.

-43 Ma L, Xu G, Sotnikova A, Szczepanowski M, Giefing M, Krause K, Krams M, Siebert R, Jin J, Klapper W: Loss of expression of lygdi (arhgdib), a rho gdp-dissociation inhibitor, in hodgkin lymphoma. British journal of haematology 2007;139:217-223.

44 Moissoglu K, McRoberts KS, Meier JA, Theodorescu D, Schwartz MA: Rho gdp dissociation inhibitor 2 suppresses metastasis via unconventional regulation of rhogtpases. Cancer Res 2009;69:2838-2844.

45 Zhang Y, Rivera Rosado LA, Moon SY, Zhang B: Silencing of d4-gdi inhibits growth and invasive behavior in mda-mb-231 cells by activation of rac-dependent p38 and jnk signaling. J Biol Chem 2009;284:1295612965.

46 Takeichi M: Cadherin cell adhesion receptors as a morphogenetic regulator. Science 1991;251:1451-1455. 